\title{
The Role of Matrix Metalloproteinase-1 and Tissue Inhibitory Matrix Metalloproteinases-3 in the Case of Bladder Neck Contracture Post Transurethral Resection of Prostate
}

\author{
Etriyel Myh ${ }^{1}$, Eryati Darwin ${ }^{1}$, Eliza Nasrul $^{1}$, Nur Rasyid ${ }^{2}$ \\ \{etriyelmyh@yahoo.com\} \\ ${ }^{1}$ Faculty of Medicine of Andalas University, Padang, Indonesia \\ ${ }^{2}$ Faculty of Medicine of Indonesia University, Jakarta, Indonesia
}

\begin{abstract}
The pathogenesis fibrosis mechanism post transurethral resection of prostate (TURP) from Bladder neck contracture (BNC) still not clear yet. Fibrosis post the wound healing is occurred by imbalanced result of breakage of extracellular matrix (ECM) by matrix metalloproteinases (MMPs). The tissue inhibitory matrix metalloproteinases (TIMPs) inhibited MMPs activity. MMP 1 and TIMP 3 play important role in the remodeling phase of wound. Aim of study to investigate role of MMP 1 and TIMP 3 in accordance to the fibrosis density on BNC post TURP. The BNC patients post TURP from M. Djamil Hospital Padang was collected. Expression of MMP 1 and TIMP 3 were measured by immunohistochemistry and fibrosis density was examined by histopathology. The $75 \%$ of samples have medium fibrosis density, $15 \%$ in the mild level and $10 \%$ in the severe level. The MMP1 expression was $27,50 \%( \pm 15,67)$ and TIMP3 was $24,94 \%( \pm 17,33)$. The correlation of expression MMP1 and fibrosis density were mild- medium with $\mathrm{R}=0,25$ and positive directional correlation $\mathrm{P}>0,0$. TIMP3 related to fibrosis density with $\mathrm{P}<0,05$ and positive directional correlation.In conclusion, TIMP3 plays significant role in fibrosis density toward BNC with positive directional correlation in post TURP.
\end{abstract}

Keywords: pathogeneis, matrix metalloproteinases, Bladder neck contracture

\section{Introduction}

Bladder neck contracture (BNC) is the fibrosis in the neck of bladder and bed of the dredging former prostate that is one of the long-term complications post TURP. The occurrence rate of BNC was $9,7 \%$ [1]. From 52 cases of BNC that were undergone re-surgeon treatment, $42 \%$ of them needed another repetition treatment and $15 \%$ needed more than twice treatments [2]. Administering Mytocimin $\mathrm{C}$ intra lesion to prevent recrudescent $\mathrm{BNC}$ was done, however the result was not effective and there was serious side effect to $7 \%$ of patients [3].

From the previous reports, there have no clarity about pathogenesis fibrosis/ BNC post TURP so that the modality of its therapy is not clear either [4]. Fibrosis is a forming of plenty extracellular matrix, which creates scar and wrecks normal architectural tissue resulting impaired organ [5]. Fibrosis is caused by the disruption of balancing between extracellular matrix formations (ECM) and its annihilation process. The formation of ECM is influenced by many growth factors [6]. 
Matrix metalloproteinase- 1 is a protease that plays a role in crushing ECM and it is proven to be a healer for remodeling scar phase [7]. MMP-1, which was founded in ureter fibrosis, was related to ureter fibrosis in ureter distal stone [8]. The role of Metalloproteinase in managing the balance between formation and destruction ECM in remodeling phase is influenced by tissue inhibitory matrix metalloproteinases (TIMPs). TIMPs consists of 4 kinds where one of them is TIMP-3 hampering all MMPs and proven in recovering scar in remodeling phase [9].

\section{Methods}

This was an observational study with cross sectional study design. The samples were taken from 20 patients who undergone second surgeon post at least 3 months treatment of TURP. Patients with the severe prostate histopathology result or BPH with exclusion prostate were the samples. BNC diagnose was conducted clinically in uretrosystoscopy. There was rigidity in the neck of bladder and there was a macroscopic fibrosis tissue in bed the dredging former prostate. Fibrosis tissue in BNC was examined by histopathology with HE staining in order to calculate fibrosis density in percentage. MMP-1 expression was investigated by immunohistochemistry (IHK) check with SC21731 antibody, MMP-1 was about $1 \mathrm{ml}$, santa cruz biotechnology -USA. In addition, the use of TIMP-3 with AB 187297 antibody, TIMP-3 was about $500 \mu \mathrm{g}$ abcam USA.

\section{Results}

From 20 samples, it can be concluded that the average age was 69,35 $\pm 7,68$ year and TURP was about 17,6 $\pm 9,10$ month. The youngest age was 54 years and the oldest one was 88 years with the condition of re- surgery treatment at least 6 months post TURP and the longest one was 36 months. The indication of re- surgery was $60 \%$ caused by the interference on LUTS and $40 \%$ caused by urinate retention. Illustration of cystoscopy BNC seen in the $1^{\text {st }}$ picture and the illustration of microscopy fibrosis density with HE staining in the $2^{\text {nd }}$ picture.
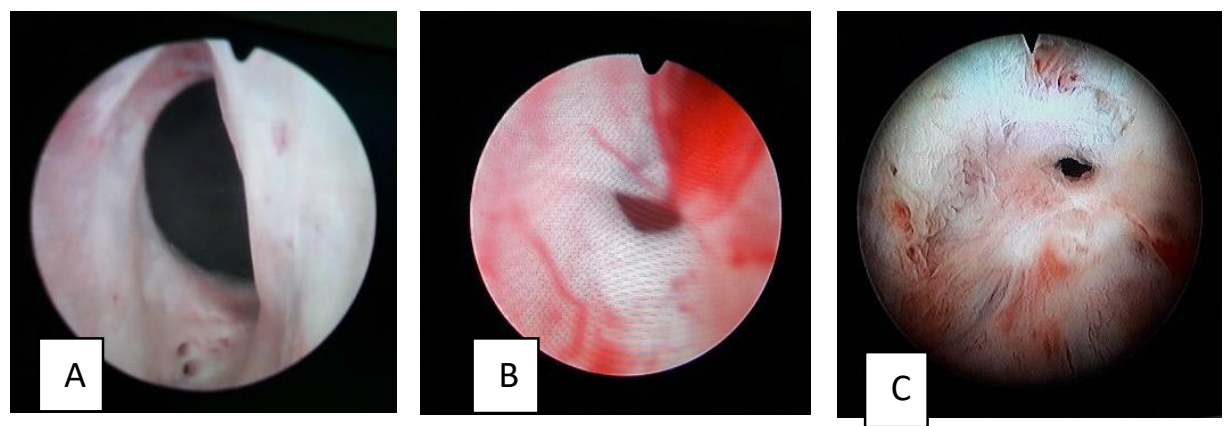

Figure 1. Illustration of Systocopy BNC A \& B is BNC with LUTS; C. BNC with urinate retention 

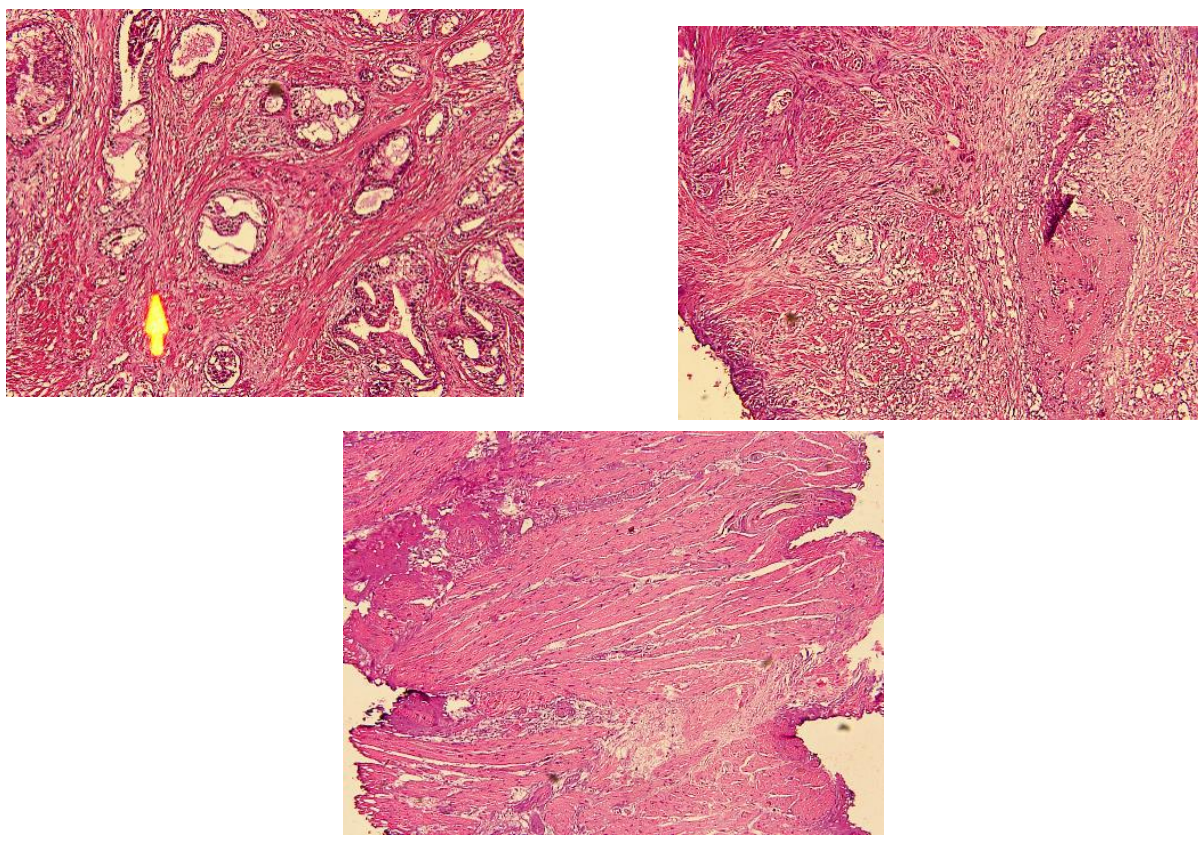

Figure 2. Illustration of Fibrosis Density with HE Staining. A. Mild Density; B. Medium Density; C. Severe Density

Fibrosis density in HE staining which is shown by Picture A presents less than $50 \%$ of fibrosis illustration meanwhile in picture B, more than 50\% fibrosis illustration yet less than $90 \%$ of fibrosis tissue. Then, in picture $C$, it is seen that more than $90 \%$ contained connective tissue.

\section{MMP- 1 Expression and TIMP-3}

The average of MMP1 was $27,50 \%( \pm 15,67)$ and TIMP3 was $24,94 \%( \pm 17,33)$. The correlation of expression MMP1 and fibrosis density were mild- medium with $\mathrm{R}=0,25$ and positive directional correlation $\mathrm{P}>0,0$. In other hand, TIMP 3 correlation and fibrosis density were medium with $\mathrm{P}<0,05$ and positive directional correlation. The illustration of MMP1 expression and TIMP3 on the IHK examination can be seen in this following picture. 


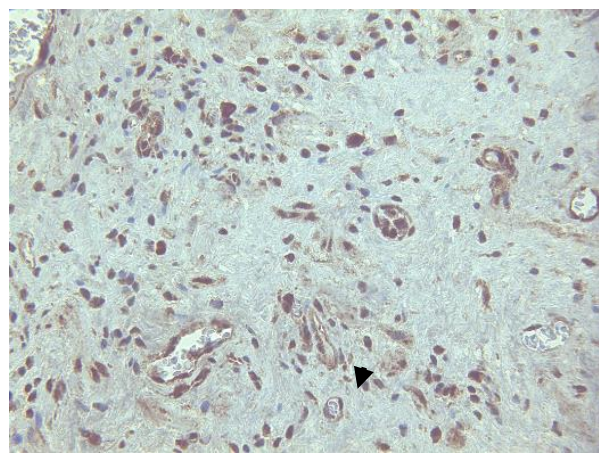

A

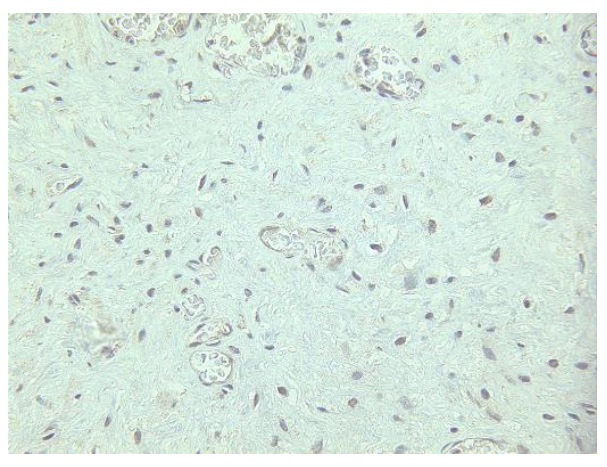

B

Figure 3. MMP1 Expression and TIMP3 on IHK Examination. A. MMP1 Expression; B. TIMP3 Expression

The Correlation between MMP1 Expression and Fibrosis Density

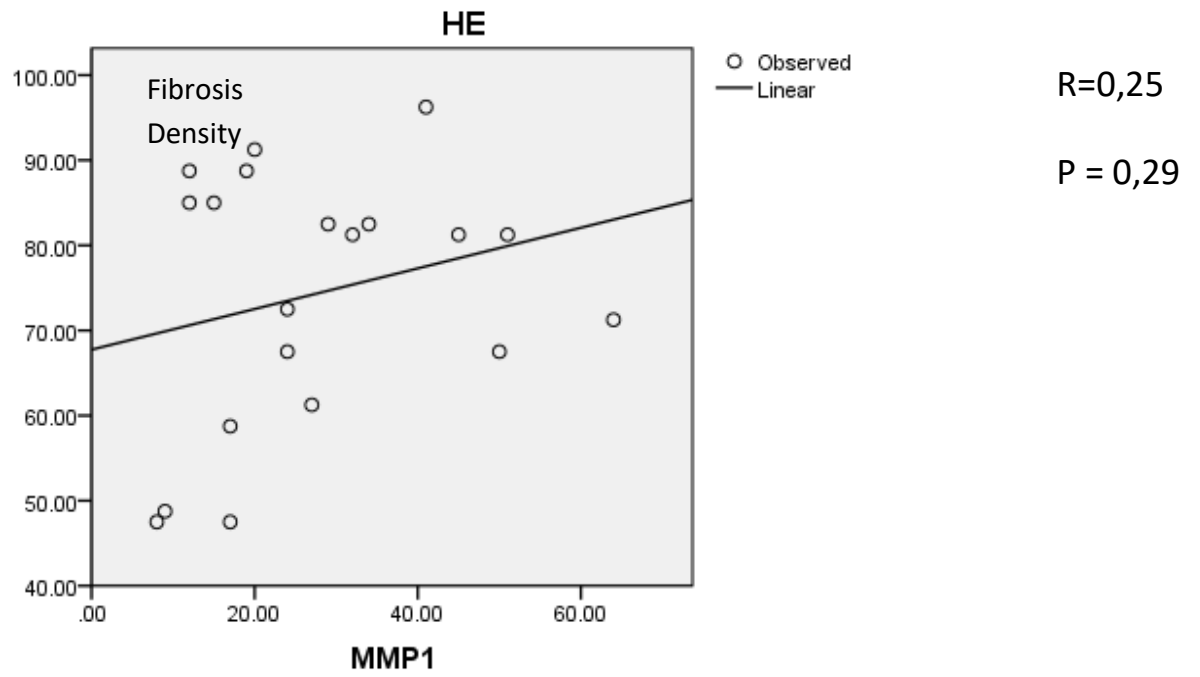

From the above picture, it can be seen that $R$ value $=0,25$ which indicates mild correlation with positive directional correlation but the significance is nothing $(\mathrm{P}>0,05)$ 


\section{Correlation between TIMP3 Expression and Fibrosis Density}

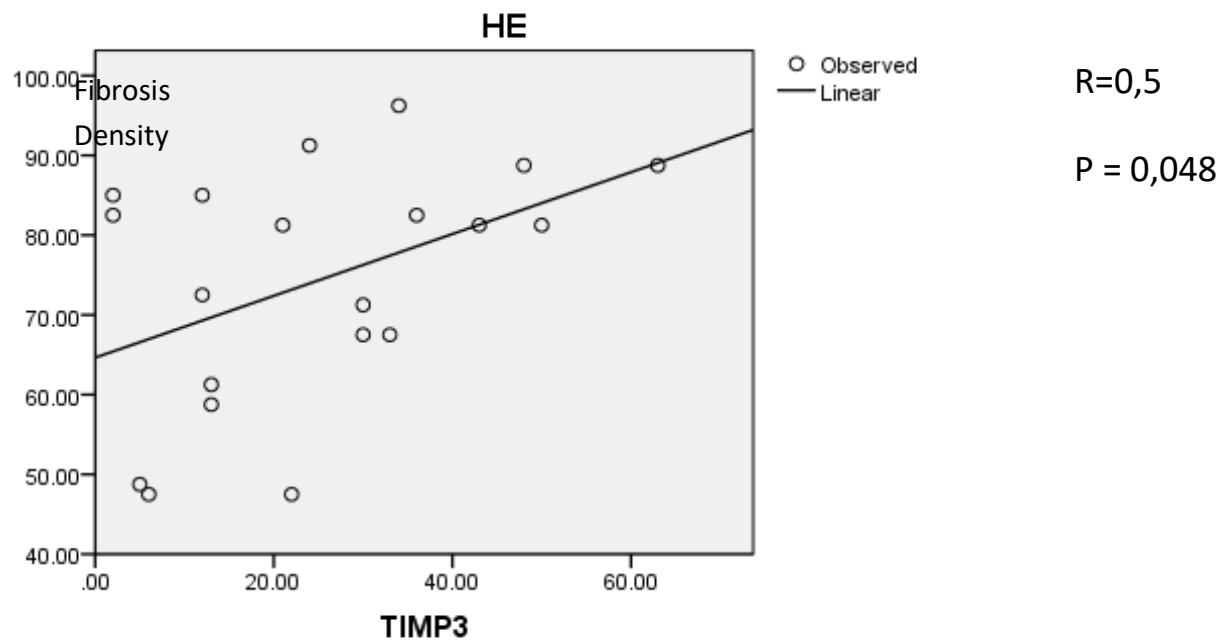

Figure 54 Correlation between TIMP3 Expression and Fibrosis Density

Based on the picture above, $\mathrm{R}$ Value is 0,5 which indicates medium correlation with positive directional correlation and the correlation is significance $(\mathrm{P}<0,05)$.

\section{Discussions}

\section{Characteristic of Sample}

The average age of patients who suffered from BNC was $69,35 \pm 7,68$. This age was more young than Al- Singary in his report about the average age of patients with BNC case that was 72,3 years [10]. From the average age of epidemiology above, it was suitable with age dispersion of BPH. Male patients in their 60 age were founded $50 \%$ BPH in biopsy and if they were in $85,90 \%$ of male patients were obtained by BPH from autopsy [11].

The occurrence`s time of BNC from TURP treatment showed the average rate 17,60 $\pm 9,10$ months, the fastest time was 6 months and the longest one was 36 months post TURP. Those results were more fast than Sata who reported the average BNC occurred in 32 months post TURP meanwhile Lee stated the average time was 37,9 months. In other hand, Al-singary conveyed the average time of BNC faster than the other two researchers that was 10,3 months. Al- singary also reported that the fastest time was 3 months and the longest was 33 months [10]. Theoretically, the times above are in the remodeling wound phase where happen around 6 months until 2 years [12]. 


\section{Fibrosis Density on HE Staining}

Determining the level of fibrosis density in organ which suffered fibrosis is one of research aspects on pathogens organ fibrosis. In liver fibrosis, there are several systems of staining such as Haematoxylin and Eosin, Reticulin and Masson's Trichrome stains. Van Gieson, Perl's and Shikata's Orcein stains. There are also several ways in determining liver fibrosis. They are Knodell Histology Activity Index (HAI), Scheuer scoring system, Ishak's system, Metavir systemdanIshak modified HAI (Mannan,2014). HAI uses some variables such as periportal bridging necrosis, intralobular degeneration and focalnerosis, portal inflammation dan fibrosis. Each of variables are given a value so that fibrosis in liver can be determined its quantitative value. Besides, the kidney fibrosis has been also identified its density fibrosis level. Related to post surgery, Sae-Jung reported that there was grading fibrosis epidural post laminectomy. Sae-Jung divided range epidural fibrosis post laminectomy into 4 grades, they are grade 0 (normal, no epidural fibrosis), grade 1 (mild degree, thin fibrous band(s) over dura), grade 2 (moderate degree, continuous adherence observed but less than $2 / 3$ of laminectomy defect) and grade 3 (severe fibrosis, scar tissue adherence, more than $2 / 3$ of laminectomy area, and/or extending to nerve roots) [14].

Unlike the case of fibrosis on BNC, we could not find the standard system in determining fibrosis density on BNC. It is because BNC is a basically a fibrosis in healing wound. Wound healing is a process to recreate normal tissue so that in normal condition the dredging former prostate after TURP in remodeling phase will be back into original prostate cell (BPH). The healing of the normal dredging prostate TURP will cause recidivism of BPH. Elshal conducted prostatectomy effectively with laser technique. He reported that there were 76 patients who are recidivism of BPH with the high average volume that was 79,3 $\mathrm{ml}$ [15]. Based on the above data, this study is done to determine the fibrosis density with measuring percentage of fibromuscular component instead the normal prostate tissue. Macroscopically, BNC had been ensured to exist in that condition.

In this study, we can sum up that most of the fibrosis density was in moderate level which means the structure of its tissue was 50-90\% consisted fibromuscular tissue component. If it is related to average time that was 17,60 $\pm 9,10$ months. This period supposed to be in the second year of healing process where it must go back to normal form of tissue.

\section{MMP1 Expression and its correlation to fibrosis density}

Expression of MMP 1 was quite high (average $27,50 \% \pm 15,67$ ) yet with statistic measurement, there was no significant correlation between MMP1 expression and fibrosis density. If it is seen from the directional correlation, it is a positive direction. The higher MMP1, the higher fibrosis density. With this result, we drawn conclusion that MMP 1 on BNC post TURP pro fibrosis.

MMP is basically anti-fibrosis, but in another condition it can be pro-fibrotic. The example is MMP 10 in recovering wounded skin. MMP 10 destroyed the scar but in term of muscle trauma, the role of MMP 10 increased collagen accumulation [16]. The transformation function of MP was caused by various growth factors such as IL-1 and TNF-Alpa, interaction among MMP where one 
MMP can be destroyed by other MMP [17] and the influence of TIMPs that can hamper MMPs performance [18].

Fibrosis is a exceeding accumulation in ECM. Collagen is the primary component of ECM. Collagen consists of many types such as collagen fibril composed. The types of this collagen are type 1,2,3,5 and 11. Membrane basal collagen that is tipe 4 collagen, micro fibril collagen that is type 7 and many others collagens [19] in every organ and organization collagen tissue forming ECM, the tissues are different that are in accordance with specific function from each organs [20].

Matrix Metalloproteinase 1 (MMP1) specifically can destroy collagen type 1 and type 3 [21], however MMP1 can not break collagen type 4 which is dominantly existed in muscle of adult person [22]. Related to this, there is a probability that collagen type 1 and 3 are not the main components of fibrosis. Nevertheless, with the destruction of collagen type 1 and 3 will make the other ECM component accumulation happened easily such as collagen type 4 so that MMP1 look like profibrotic. The activity of MMP1 above was proven in experimental of Duchenne muscular dystrophy (DMD). Giving MMP 1 has an ability to wreck the scar in muscle which was infected by DMD. It also assists in increasing myoblast migration so that muscle fiber in DMD case enhanced [19].

\section{TIMP 3 Expression and its correlation with fibrosis density}

Tissue inhibitory matrix metalloproteinases 3 (TIMP 3) is a protein hampering MMP performance so that MMP is not applied to wreck the scar. The mechanism of obstructing MMP activity by TIMP occurred because TIMP ties up MMP and produces TIMP- MMP complex. The molecule form of TIMP is steel and has 4 residues in its terminal. They are Cys1-Thr-Cys-Val4 and Glu67-Ser-Val-Cys70 as residue which related to disulfide in active side of MMP. Actually, the bounding or correlation between MMP and TIMP is relationship of protein and other protein. Mutation in second position from residue $\mathrm{N}$ molecule terminal TIMP influenced the relationship afinitas TIMP and MMP. If the position were change by glysin impacted on non active TIMP. In TIMP 3, if the mutation in Thr 2 becomes Gly will affect to non active of TIMP3 [23].

Tissue inhibitory matrix metalloproteinases 3 (TIMP3) is a main regulator from MMP invivo. The percentage of TIMP 3 was the lowest rather than MMP-1 and TGF $\beta 1$. However, if it is connected to fibrosis density, there was significant connection to TIMP3 with positive directional connection. It means the more high TIMP3 expression, the more high fibrosis density. This condition is suitable with TIMP3 physiology as protein which confronts MMP performance to lysis fibrosis tissue. When the obstruction of MMP performance happened, it will increase the accumulation of fibrosis tissue. The final result of fibrosis accumulation in a tissue is the result of difference of fibrosis tissue that is divided by MMP and it is minimized by total hindrance of TIMPs [18].

So, from this study, we can get the fact that MMP1 is pro-fibrotic. With this condition, there is a probability of another MMP besides MMP1 which play role to ruin main component ECM creating fibrosis in BNC which hampered by TIMP3. In the term of urethra diseases, there are several researches about it, for example the role of TIMP3 and its connection to MMP2 and MMP9 causing 
the increase of risk of Buli- Buli cancer (carcinoma buli- buli) [24]. Specifically, TIMP3 hampered MMP 1,2,3,9 and 13 [25]. Thus, there is a possibility that the hampering TIMP3 which makes the improvement of fibrosis density related to MMP other than the stated above.

Therefore, after conducting this study, it can be founded that the role of TIMP 3 is a significant variable. In fact, TIMP 3 can be used as one of therapy modality for BNC case by developing anti TIMP 3. Manipulation of TIMP 3 as one of therapy modality has also been examined into cardiovascular disease and its terminal effect. Administering TIMPs for gastric cancer has reached clinical examination into phase 3 and phase 2 toward pancreatic cancer [26].

\section{Conclusion}

MMP 1 and TIMP 3 are expressed in BNC post TURP. They are related to fibrosis density so that TIMP 3 becomes significant factor in the BNC case. The higher TIMP 3 , the denser fibrosis in BNC case post TURP.

\section{REFERENCES}

Lee YH, Chiu AW, Huang JK. 2005. Comprehensiv study of bladder neck contracture after transurethral resection of prostate. Urology. 65: 498-503.

Borboroglu PG, Sands JP, Roberts JL, Amling CL. 2000. Risk factors for vesico urethral anastomotic stricture after radical prostatectomy.Urology.56:96-100.

Roehborn CG, Redshaw J, Broghammer JA, Smith TG, Voelzke BB, Erickson BA, et al, 2014. Intralesional Injection of Mitomycin $\mathrm{C}$ at Transurethral Incision of Bladder Neck Contracture May Offer Limited Benefit: from the TURNS Study Group . J. Jurol.103: 1-2.

Moldoveanu C, Geavlete B, Stănescu F, Jecu M, Adou L, Bulai C, et al. 2013. Tips and Tricks in Secondary Bladder neck sclerosis bipolar plasma vaporization approach. J Med Life. 6: 27277.

Agarwal KS. 2014. Integrin and cadherin as therapeutic targets in fibrosis. Front Pharmacol. 5: 131. Bellayr IH, Walter TJ, Li Y.2010. Scarless wound healing. J Am Coll Clin Wound Spec. 2: 40-3.

Brenneisen P, Wenk J, Wlaschek M, Krieg T, Scharffetter-Kochanek K. 2000. Activation of p70 ribosomal protein S6 kinase isan essential step in the DNA damage-dependent signaling pathway responsible for the ultraviolet B-mediated increase in interstitial collagenase (MMP1) and stromelysin-1 (MMP-3) protein levels in human dermal fibroblasts. J Biol Chem. 275:4336-44.

Zuhirman, Yanwirasti, Rasyid.2014. Hubungan Transforming Growt Factor $\beta$ 1, Tumor Necrosis Factor- $\alpha$, Matrix Metalloproteinase-1 dan Fibroblast Growth Factor -2 dengan fibrosis Ureter padaPenderitaBatu Ureter.Disertasi. UniversitasAndalas. Padang

Gill SE, Parks W. 2008. Metalloproteinases and Their Inhibitors: Regulators of Wound Healing. Int J Biochem Cell Biol. 40(6-7): 1334-47 
Al-Singary W, Arya M, Patel HR. 2004. Bladder neck stenosis after transurethral resection of prostate : does size matter?. Urol Int.73: 262-5.

Kellerman, Rick D. 2015. Conn's Current Therapy 2015. Saunders Goldman L, Schafer A I. Benign Prostate Hyperplasia and Prostatis. In: Goldman's Cecil Medicine. 24 ${ }^{\text {th }}$ Ed. Saunders 2012. 805-10.

Pakyari M, Farrokhi A, Maharlooei MK, Ghahary A. 2013. Critical Role of transforming growth factor beta in different phases of wound healing. Adv Wound care.2 (5):215-24.

Mannan R, Misra V, Misra SP, Singh PA, Dwivedi M. 2014. A Commaprative evaluation of scorring system for assessing necro inflammatory activity and fibrosis in liver biopsies of patients with chronic viral hepatitis. J cllin Diagn Res.8(8):8-12.

Sae -jung S, Jirarattanaphocha K, Sumananont C, Wittayapairoj K,Sukhonthamarn K. 2015. Interrater Reliability of the Postoperative Epidural Fibrosis Classification: A Histopathologic Study in the Rat Model.Asian Spine J. 9(4): 587-594.

Elshal AM, Elkoushy MA, El-Nahas AR, Shoma AM, Nabeeh A, Carrier S, et al. 2015. GreenLight $^{\mathrm{TM}}$ laser (XPS) photoselective vapo-enucleation versus holmium laser enucleation of the prostate for the treatment of symptomatic benign prostate hyperplasia: a randomized controlled study. J Urol. 193(3):927-34.

Bobadila M, Sainz N, Rodriguez JA, Abizanda G, Orbe J, deMartino A, et al. 2014. MMP-10 is required for efficient muscle regeneration in mouse models of injury and muscular dystrophy. Stem cells. 32:447-61.

Amaleinei C, Irina DrogaCarutu, RalucaAnca Balan.2007. Biology of Metalloproteases and remodelin. Rom J Morphol Embryol. 48(4):323-34.

Matthew G and Parks WC. 2014. Diverse functions of matrix metalloproteinases during fibrosis. Disease Models \& Mechanisms. 7;193-203.

Pan H, Vojnits K, Liu TT, Meng F, Yang L, Wang Y, et al. 2015. MMP1 gene expression enhances myoblast migration and engraftment following implanting into mdx/SCID mice. Cell Adhesion \& Migration, 9:4, 283-92.

Cox TR, Erler JT. 2011. Remodeling and homeostasis of the extracellular matrix: Implication for fibrotic disease and cancer. Disease Models \& mechanism.4:165-78.

Lauer-Fields JL, Juska D, Fields GB. 2002. Matrix metalloproteinases and collagen catabolism. Biopolymers.PMID:12228918; http://dx.doi.org/ 10.1002/bip.10201. 66:19-32;

Ahtikoski AM, Koskinen SO, Virtanen P, Kovanen V, Risteli J, Takala TE. 2003. Synthesis and degradation of type IV collagen in rat skeletal muscle during immobilization in shortened and lengthened positions. Acta Physiol Scand; 177:473-81.

Nagase H, VisseR, Murphy G. 2006. Structure and function of Matrix metalloproteinases and TIMPs.Cardiovascular Research.69:562-73.

Wieczorek E, Reszka E, Jablonowski Z, Jabloska E, Krol MB, et al. 2013. BJU Int.112:1207-14. 
Vanhautte D, Heymans S. 2009. TIMPs and cardiac remodeling:'Embracing the MMP-independentside of the family'. J Mol Cell Cardiol.

Baker AH, Edwards DR, Murphy G. 2002. Metalloproteinase inhibitors: Biological actions and therapeutic opportunities. J Cell Sci.115:3719-27. 